\title{
Reconhecimento dos padrões eletrocardiográficos equivalentes de infarto agudo do miocárdio com supradesnivelamento de segmento ST
}

\author{
Importance of recognizing equivalent electrocardiographic patterns of acute myocardial \\ infarction with ST segment elevation: an integrative review
}

Importancia de reconocer patrones electrocardiográficos equivalentes de infarto agudo de miocardio con elevación del segmento ST: una revisión integradora

Angelo Bruno Pagoto ${ }^{1 *}$, Carlos Roberto Rohenkohl Evangelista Santos ${ }^{1}$, Felipe Augusto Ferreira Vitorio ${ }^{1}$, Marlucia do Nascimento Nobre ${ }^{1}$, Rovanda Guimarães Sena ${ }^{1}$, Irineu Lopes de Alcântara Júnior ${ }^{1}$, Armando Hiroyuki Mori Júnior ${ }^{1}$, Bárbara Pires Ihara ${ }^{1}$.

\begin{abstract}
RESUMO
Objetivo: Avaliar o que as publicações têm tratado sobre a importância de reconhecer precocemente diferentes padrões eletrocardiográficos, mais especificamente os equivalentes de Infarto Agudo do Miocárdio Com Supra do segmento ST (IAMCSST/STEMI). Métodos: Trata-se de uma revisão integrativa, com recorte temporal entre 2010 e 2020. Para elaboração da revisão foi estabelecida questão norteadora: "qual a importância do reconhecimento dos padrões eletrocardiográficos equivalentes de IAMCSST? ". Para busca de dados foram usadas Biblioteca Virtual em Saúde e Pubmed e foram associados aos descritores em português e inglês "ST Elevation Myocardial Infarction equivalent", "Electrocardiography" e "Electrocardiography patterns". Para coleta e análise excluiu-se artigos repetidos, com posterior leitura íntegra. Resultados: A partir da análise das 22 publicações selecionadas, reconhece-se que a avaliação dos diferentes padrões eletrocardiográficos estão aquém do considerado ideal, o tempo hábil na pronta interpretação de exame é tardio e as mudanças dos equivalentes de IAMCSST demonstraram-se como alterações sutis que passam despercebidas por profissionais inexperientes. Considerações finais: Os padrões equivalentes STEMI são pouco reconhecidos na interpretação do ECG e apresentam em sua maioria alta taxa de mortalidade associada a um prognóstico ruim. É necessário que o profissional de saúde tenha acesso a informação dos desenhos eletrocardiográficos de maneira integral.
\end{abstract}

Palavras-chave: Infarto do miocárdio com supradesnível do segmento ST, Eletrocardiografia, Infarto do miocárdio.

\begin{abstract}
Objective: To evaluate what publications have been dealing with about the importance of early recognition of different electrocardiographic patterns, more specifically the equivalents of ST-segment Supra Acute Myocardial Infarction (IAMCSST/STEMI). Methods: This is an integrative review, with a time frame between 2010 and 2020. To prepare the review, a guiding question was established: "what is the importance of recognizing the equivalent electrocardiographic patterns of STEMI?". For data search, the Virtual Health Library and Pubmed were used and they were associated with the descriptors in Portuguese and English "ST Elevation Myocardial Infarction equivalent", "Electrocardiography" and "Electrocardiography patterns". For collection and analysis, repeated articles were excluded, with subsequent full reading. Results: From the analysis of the 22 selected publications, it is recognized that the evaluation of the different electrocardiographic patterns are below what is considered ideal, the time for prompt interpretation of the exam is late and the changes in the STEMI equivalents were shown to be subtle changes that go unnoticed by inexperienced professionals. Final considerations: STEMI equivalent patterns are poorly recognized in ECG interpretation and mostly present a high mortality rate associated with a poor prognosis. It is necessary that the health professional has access to information on electrocardiographic drawings in full.
\end{abstract}

Keywords: ST elevation myocardial infarction, Electrocardiography, Myocardial infarction.

1 Universidade Federal do Amazonas (UFAM), Manaus - AM. *E-mail: angelo@pagoto.com.br 


\section{RESUMEN}

Objetivo: Evaluar qué publicaciones han abordado sobre la importancia del reconocimiento temprano de diferentes patrones electrocardiográficos, más específicamente los equivalentes del infarto de miocardio supraagudo del segmento ST (IAMCSST / STEMI). Métodos: Se trata de una revisión integradora, con un período de tiempo comprendido entre 2010 y 2020. Para la elaboración de la revisión se estableció una pregunta orientadora: " ¿Cuál es la importancia de reconocer los patrones electrocardiográficos equivalentes del IAMCEST?". Para la búsqueda de datos se utilizó la Biblioteca Virtual en Salud y Pubmed y se asociaron a los descriptores en portugués e inglés "Equivalente de infarto de miocardio con elevación del ST", "Electrocardiografía" y "Patrones de electrocardiografía". Para la recopilación y el análisis, se excluyeron los artículos repetidos, con posterior lectura completa. Resultados: Del análisis de las 22 publicaciones seleccionadas, se reconoce que la evaluación de los diferentes patrones electrocardiográficos está por debajo de lo que se considera ideal, el tiempo para la pronta interpretación del examen es tardío y se demostró que los cambios en los equivalentes de STEMI son sutiles cambios que pasan desapercibidos para profesionales sin experiencia. Consideraciones finales: Los patrones equivalentes de STEMI son poco reconocidos en la interpretación del ECG y en su mayoría presentan una alta tasa de mortalidad asociada con un mal pronóstico. Es necesario que el profesional de la salud tenga acceso a la información de los dibujos electrocardiográficos en su totalidad.

Palabras clave: Infarto del miocardio con elevación del ST, Electrocardiografía, Infarto del miocardio.

\section{INTRODUÇÃO}

A Síndrome Coronariana Aguda (SCA) tem sua causa na obstrução das artérias coronarianas, que irrigam o músculo do coração, devido à interação dos fenômenos de trombose e vasoespasmos, resultando em sinais clínicos como angina, irrradiação da dor para o membro superior esquerdo, sincope e até mesmo óbito, caso não haja uma intervenção médica. Alguns dos fatores de risco mais prevalentes são o tabagismo, dislipidemia, história familiar pregressa da doença, idade avançada, sexo masculino e diagnóstico de Diabetes Mellitus (SILVA LN, et al., 2018).

A SCA segue no contexto da atualidade como uma das maiores causas de morbimortalidade mundial, sendo os fatores cardíacos de uma maneira geral os que configuram como a doença com a maior taxa de óbito dentre todas as outras. Dito isso em um cenário de urgência e emergência, tornam-se necessárias ferramentas de fácil acesso e aplicabilidade com uma ampla distribuição e munidas de rapidez diagnóstica. O eletrocardiograma (ECG), por reunir as características desejadas, segue sendo o exame mais importante nesse contexto. Sua importância baseia-se no fato de que pelas atuais diretrizes ele é o definidor da administração de trombolítico ou intervenção percutânea primária na vigência do infarto transmural. Como é amplamente sabido, a expressão eletrocardiográfica desse quadro é o supradesnivelamento do segmento ST (TZIMAS G, et al., 2019).

A literatura sugere que pode haver algum tipo de erro na interpretação do ECG por parte dos médicos clínico e especialista em até $33 \%$ e $11 \%$, respectivamente, evidenciando a existência de um grande déficit entre os clínicos de maneira geral e também uma parcela menor, ou seja, menos significativa, porém ainda existente de erros dentre os especialistas, ficando a conclusão de que o viés e a dificuldade de interpretar corretamente um ECG não assombra apenas os médicos menos experientes, mas também os detentores de maior maestria técnica (BREEN CJ, et al., 2019).

No mais, Bond RR (2014) demonstrou em um grupo de cardiologistas uma confiabilidade interexaminadores de $79 \%$ para diagnosticar um quadro de infarto agudo do miocárdio com supradesnivelamento do segmento ST (IAMCSST ou STEMI em inglês), um valor que pode ser considerado como questionável uma vez que por se tratarem de expertises tal porcentagem deveria margear mais próximo dos $100 \%$ considerando obviamente de forma otimista um cenário ideal (TZIMAS G, et al., 2019).

Nos últimos 10 anos, uma parcela significativa de trabalhos têm demonstrado a existência de padrões eletrocardiográficos presumíveis de infarto tão importantes quanto o supradesnivelamento do segmento ST (STEMI), esses modelos configuram-se na literatura em 5 grupos diversos que possuem semelhança entre si 
principalmente quando se trata da comparação entre as grafias características do ECG, são eles: o bloqueio de ramo esquerdo (BRE), a síndrome de Wellens, o sinal de Winter, o chamado supra de AVR e o bloqueio de ramo direito (BRD) (MACIAS M, et al., 2016).

Obviamente, pela importância que esses achados trazem para a condução dos pacientes, 0 reconhecimento dos mesmos torna-se importantíssimo para uma maior celeridade e correta condução dos casos. De fato, tais registros têm ganhado tanta atenção, que começam a ser denominados pela literatura de "equivalentes do Infarto Agudo do Miocárdio com Supradesnivelamento do Segmento ST" ou simplesmente "equivalentes IAMCSST" (ZHAN ZQ, et al., 2020).

O BRE é sem dúvida o mais conhecido, constando em todas as diretrizes de IAMCSST como American Heart Association (AHA) (2015), European Society of Cardiology (ESC) (2016) e Sociedade brasileira de Cardiologia (SBC) (2020), além disso é elucidado na literatura científica que com o decorrer dos últimos anos têm-se evidenciado o quanto são comuns outros padrões de equivalentes de IAMCSST. Nesse ínterim, a literatura também indica que de 10 a $25 \%$ dos casos apresentavam esse padrão, com necessidade de terapia de reperfusão de urgência, concluindo que os guidelines futuros deveriam incorporar tais alterações para maior conhecimento da comunidade médica (WALL J, et al., 2018).

De Zwaan C, et al. (1982) demonstrou que $75 \%$ dos pacientes com síndrome de Wellens que não foram abordados, evoluíram com infarto anterior extenso em um período de poucas semanas. Em um grande estudo de coorte com 5588 pacientes, Winter RW, et al. (2019) detectou que 1,6\% dos quadros de infarto anterior tinham um padrão com mortalidade de $27 \%(n=3)$.

Além dos exemplos anteriores, existem outros padrões que guardam correlação, em termos de gravidade, com o supradesnivelamento do segmento ST. Grande parte deles não são descritos nas diretrizes, mas aceitos e reconhecidos por sociedades de cardiologia, como é o caso do supra de AVR (por vezes denominada de derivação orfã), onda T hiperaguda, bloqueio de ramo esquerdo, bloqueio de ramo direito, dentre outros (BREEN CJ, et al., 2019).

Ao constatar que o paciente apresentou infarto com elevação de ST necessita ser avaliado de forma rápida e precisa. É de informação geral da área médica que atrasos na conduta de reperfusão desses pacientes aumentam muito a taxa de mortalidade, além disso a lentidão é fator diretamente proporcional ao mal prognóstico. As diretrizes atuais exigem que a interpretação do ECG tenha tempo máximo de 10 min após a chegada do paciente, no entanto, muitos deles não recebem reperfusão a tempo justamente porque há desafios na interpretação dos padrões (MCLAREN JTT, et al., 2020).

Essas dificuldades ocorrem devido primeiro a conformidade dos profissionais em não reavaliar interpretações quando são automatizadas, uma vez que essas possuem altas taxas de erros, por isso necessitam ser sempre confirmadas, segundo a identificação de padrões equivalentes STEMI que passam despercebidos, nesse sentido há um número de oclusões coronárias agudas que são perdidas e consequentemente atraso e prejuízo na ativação do laboratório de cateterismo desses pacientes (YANG W, et al., 2017).

Dessa forma, é possível perceber a importância da avaliação criteriosa dos padrões eletrocardiográficos e fornecer embasamento científico para os profissionais utilizarem das melhores práticas no momento do tratamento e manejo clínico. Portanto, tem-se como objetivo avaliar, por meio da literatura científica, o que as publicações têm tratado sobre a importância de reconhecer precocemente esses diferentes padrões eletrocardiográficos, mais especificamente os equivalentes de IAMCSST (STEMI).

\section{MÉTODOS}

Esta pesquisa é uma revisão integrativa da literatura científica, a qual baseia-se na busca de conhecimento e na análise crítica sobre importantes temas no contexto de saúde. Foi determinado como recorte temporal para a seleção das produções bibliográficas o período correspondente entre 2010 e 2020 e adotou-se as etapas propostas e reproduzidas por Souza MT, et al. (2010) para a elaboração da revisão, que consistem na elaboração da pergunta norteadora, amostragem na literatura, coleta de dados, análise crítica dos estudos, discussão dos resultados e apresentação da revisão. 
Inicialmente, foi estabelecido a questão norteadora da pesquisa: "Qual a importância do reconhecimento dos padrões eletrocardiográficos equivalentes de infarto agudo do miocárdio com supradesnivelamento de segmento ST?". Em seguida, realizou-se a busca na literatura científica por meio das bases de dados Biblioteca Virtual em Saúde (BVS) e PubMed utilizando descritores, em português e inglês, combinados entre si com os operadores booleanos AND e OR. Os descritores utilizados foram: "ST Elevation Myocardial Infarction equivalent", "Electrocardiography" e "Electrocardiographic patterns".

Para a coleta e análise crítica dos dados, verificou-se o título e o resumo das publicações e determinouse critérios de inclusão e exclusão, sendo adotados como critérios de inclusão: artigos publicados em língua portuguesa, inglês ou espanhola, disponíveis na íntegra, condizentes com o recorte temporal préestabelecido. Escolheu-se como critério de exclusão: artigos repetidos por intercessão nas plataformas de pesquisa, artigos que tangenciavam a temática da pesquisa, teses, dissertações e trabalhos de conclusão de curso. Ademais, a partir dessa seleção, realizou-se a leitura minuciosa dos artigos discriminados na íntegra.

As informações extraídas das publicações foram organizadas em forma de quadro, por intermédio do software Microsoft Excel® 2016, o qual foi estruturada em autores, ano de publicação, tipo de pesquisa e principais achados. Dessa maneira, posteriormente foi abordado e discutido de forma coesa com sobre a temática central do estudo.

\section{RESULTADOS}

Realizou-se a procura dos artigos nas bases de dados citadas e foram encontradas 1.800 publicações referenciando os descritores selecionados de acordo com a Figura 1, dentre os quais, 1.557 foram da Biblioteca Virtual em Saúde (BVS) e 243 da PubMed. Posteriormente, adotou-se os critérios de inclusão e foram selecionadas 273 produções, das quais 231 foram removidas por repetirem em diferentes plataformas ou por não serem compatíveis com a temática central da pesquisa. Das 42 publicações restantes, após leitura minuciosa na íntegra, foram selecionados, portanto, 22 artigos para processamento, análise e discussão de resultados.

Figura 1 - Fluxograma de pesquisa nas bases de dados.

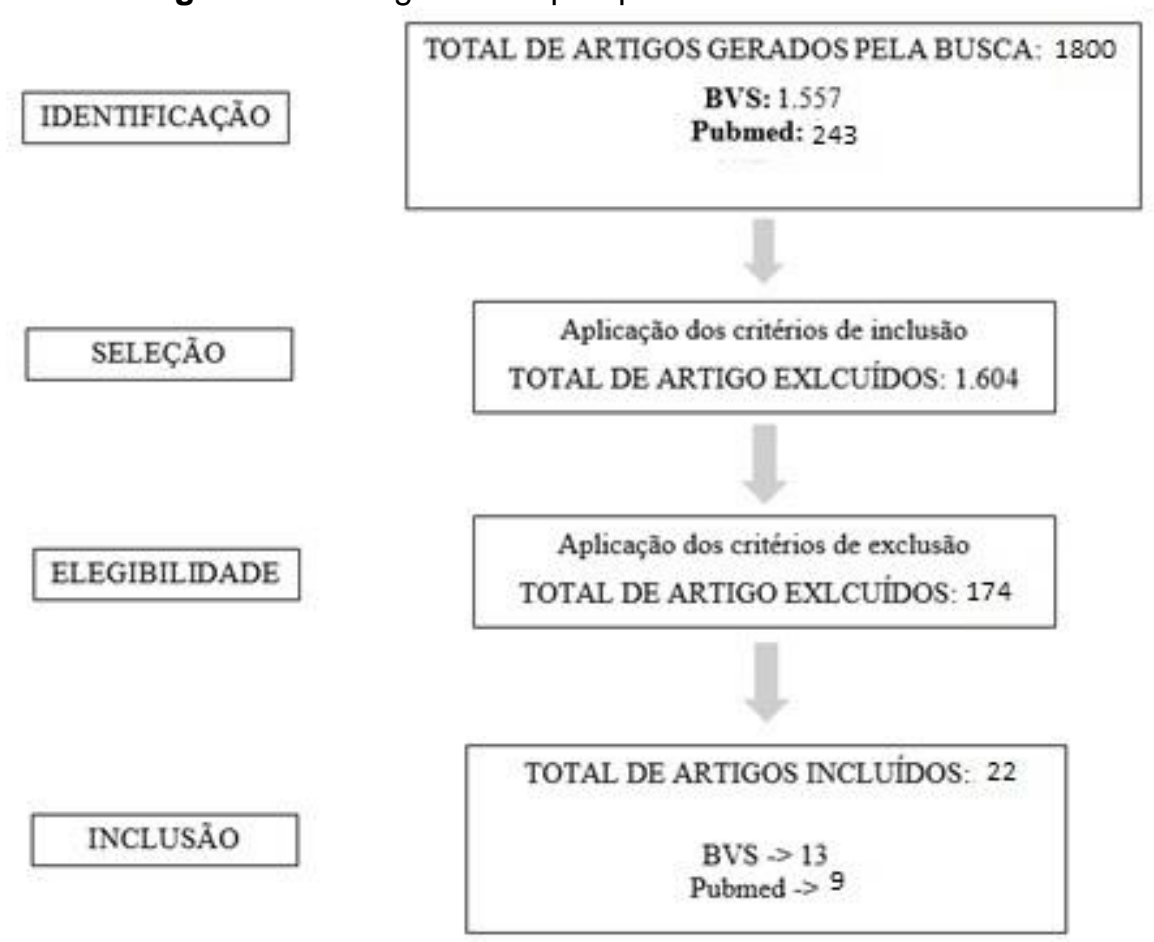

Fonte: Pagoto AB, et al., 2021.

O Quadro 1 ilustra as características dos estudos selecionados, contendo os autores, o ano de publicação, o método utilizado e os principais achados. 
Quadro 1 - Síntese dos principais achados sobre a temática.

\begin{tabular}{|c|c|c|}
\hline $\mathbf{N}$ & Autores (Ano) & Principais achados \\
\hline 1 & BOSSON N, et al. (2016) & $\begin{array}{l}\text { Série de casos. Foram analisados } 44.611 \text { ECG's com intuito de classificar a interpretação dos padrões encontrados; } \\
\text { As principais causas de infarto (STEMI ou não) falso-positivos foram pela interpretação do ECG ou por causas não } \\
\text { isquêmicas de supra de ST. }\end{array}$ \\
\hline 2 & SEFA N e SAWYER KN (2016) & $\begin{array}{l}\text { Relato de caso. Caso de um homem de 84anos internado, em que foi diagnosticado um quadro de STEMI pelos } \\
\text { critérios de Sgarbossa modificados por Smith; É fundamental que o médico de emergência conheça tais critérios para } \\
\text { que o ambiente clínico torne-se mais apropriado. }\end{array}$ \\
\hline 3 & CARR MJ, et al. (2016) & $\begin{array}{l}\text { Relato de caso. O paciente de } 33 \text { anos foi identificado com um padrão eletrocardiográfico de Winter; É fundamental } \\
\text { que o médico de emergência aprenda e saiba identificar esse padrão de ECG equivalente de STEMI para garantir a } \\
\text { adequada intervenção ao paciente. }\end{array}$ \\
\hline 4 & MACIAS M, et al. (2016) & $\begin{array}{l}\text { Revisão bibliográfica. Aborda cinco padrões de ECG equivalentes a STEMI: IAM de parede posterior, Síndrome de } \\
\text { Wellens, sinal de Winter, } 1^{\circ} \text { ramo diagonal da LAD e oclusão da LMCA; É imprescindível que os médicos de } \\
\text { emergência reconheçam esses padrões. }\end{array}$ \\
\hline 5 & HANNA EB, et al. (2015) & $\begin{array}{l}\text { Série de casos. Visa apresentar que nem sempre a suspeita de SCA ou BRE implica em um STEMI; Ilustra que } \\
\text { apenas uma minoria dos pacientes suspeitos tem um ECG equivalente de STEMI. }\end{array}$ \\
\hline 6 & PAHLM U, et al. (2014) & $\begin{array}{l}\text { Revisão bibliográfica. Avalia a determinação de STEMI a partir do ECG de } 12 \text { derivações e a necessidade de } \\
\text { maximiza-lo; O uso do ECG de } 24 \text { derivações melhoraria a capacidade de reconhecer diferentes padrões e facilitar } \\
\text { o tratamento médico. }\end{array}$ \\
\hline 7 & AYER A e TERKELSEN CJ (2014) & $\begin{array}{l}\text { Revisão bibliográfica. Analisa alguns exemplos críticos de padrões de ECG que geralmente são mal interpretados ou } \\
\text { não identificados; Os equivalentes de STEMI são frequentemente associados a atrasos no tratamento do paciente e } \\
\text { pior resultado. }\end{array}$ \\
\hline 8 & WEI EY, et al. (2013) & $\begin{array}{l}\text { Estudo de coorte. Avalia a incidência de pacientes realmente com STEMI e a perda do padrão ao longo de } 11 \text { meses; } \\
\text { Os equivalentes de STEMI, atualmente, prejudicam o diagnóstico e são desafio diagnóstico significativo. }\end{array}$ \\
\hline 9 & LAWNER SM, et al. (2012) & $\begin{array}{l}\text { Revisão bibliográfica. Analisa exemplos de padrões de ECG equivalentes de STEMI: complexo de Winter, Síndrome } \\
\text { de Wellens, infarto de parede posterior e alterações advindas de BRE. O diagnóstico de STEMI é considerado } \\
\text { sensível ao tempo e necessita de maior conhecimento pelos médicos. }\end{array}$ \\
\hline 10 & HARTMAN SM, et al. (2012) & $\begin{array}{l}\text { Artigo original. Foi desenvolvido um algoritmo de } 4 \text { que visa detectar STEMI na maioria das situações pré-hospitalares } \\
\text { ou de emergência. Este método facilita a interpretação por parte de profissionais inexperientes, porém, pode não } \\
\text { detectar corretamente padrões equivalentes. }\end{array}$ \\
\hline 11 & BREEN CJ, et al. (2019) & $\begin{array}{l}\text { Revisão bibliográfica. Avalia a aquisição de habilidades na interpretação de ECG. O estabelecimento de uma } \\
\text { interpretação universal, o treinamento do corpo docente e o formato do ensino variam muito de localidade, e } \\
\text { prejudicam diretamente na interpretação de diferentes padrões. }\end{array}$ \\
\hline
\end{tabular}




\begin{tabular}{|c|c|c|}
\hline $\mathbf{N}$ & Autores (Ano) & Principais achados \\
\hline 12 & WINTER RW, et al. (2016) & $\begin{array}{l}\text { Relato de caso. Relato de três pacientes com sintomas típicos de IAM; visando uma oportuna terapia de reperfusão, } \\
\text { é importante que o reconhecimento de equivalentes de STEMI seja garantido pela equipe envolvida nas situações de } \\
\text { emergência. }\end{array}$ \\
\hline 13 & HANNA EB, et al. (2015) & $\begin{array}{l}\text { Relato de caso. Apresentado um padrão de ECG descrito por Winter et al; llustra um padrão que apresenta } \\
\text { características do descrito por Winter, mas que parece ser diferente do originalmente descrito. }\end{array}$ \\
\hline 14 & BOND RR, et al. (2014) & $\begin{array}{l}\text { Artigo original. Avalia uma tecnologia de rastreamento ocular para obter informações sobre a interpretação do ECG } \\
\text { de } 12 \text { derivações; recomenda-se que os interpretadores adotem um protocolo sistemático de observação, visando } \\
\text { reduzir os diagnósticos de primeira impressão }\end{array}$ \\
\hline 15 & MCLAREN JTT, et al. (2020) & $\begin{array}{l}\text { Estudo retrospectivo. Avalia o tempo para determinar o diagnóstico de oclusões coronarianas por médicos de } \\
\text { emergência utilizando o ECG; O tempo de ativação de ECG (ETA) pode servir como um parâmetro de qualidade, } \\
\text { principalmente nos critérios de STEMI e equivalentes. }\end{array}$ \\
\hline 16 & MUHLESTEIN JB, et al. (2020) & $\begin{array}{l}\text { Ensaio clínico não randomizado. Avalia a viabilidade da combinação de ECG seriados de eletrodo único em } \\
\text { smartphone. Essa tecnologia apresenta uma boa correlação de resultado com o ECG de } 12 \text { derivações, sendo uma } \\
\text { futura alternativa para tratamento precoce de STEMI. }\end{array}$ \\
\hline 17 & PERA VK, et al. (2018) & $\begin{array}{l}\text { Artigo original. Analisa características de pacientes STEMI e testa um novo algoritmo de triagem para diagnosticar } \\
\text { pacientes com BRE; se validado, o novo algoritmo pode melhoras a precisão do diagnóstico em relação a terapia de } \\
\text { reperfusão. }\end{array}$ \\
\hline 18 & RAJA JM, et al. (2019) & $\begin{array}{l}\text { Revisão sistemática. Aborda a importância da detecção do sinal de De Winter e a urgência de intervenção. A } \\
\text { evidência sugere que o sinal, apresentando depressão em ST e elevação da onda T, deve ser tratado como } \\
\text { equivalente de STEMI, com reconhecimento e intervenção precoce. }\end{array}$ \\
\hline 19 & TZIMAS G, et al. (2019) & $\begin{array}{l}\text { Revisão bibliográfica. Analisa os equivalentes de STEMI: Síndrome de Wellens, sinal de Winter, BRE, BRD e ondas } \\
\text { T hiperagudas. O reconhecimento desses equivalentes é crucial para todo profissional que lida com pacientes com } \\
\text { SCA no departamento de emergência. }\end{array}$ \\
\hline 20 & WISNIEWSKI P, et al. (2019) & $\begin{array}{l}\text { Ensaio clínico. Visa identificar o valor preditivo dos padrões equivalentes de STEMI para detectar oclusão em } \\
\text { pacientes NSTEMI. Os equivalentes não parecem ter uma vantagem relevante sobre as alterações isquêmicas } \\
\text { clássicas de ECG na predição de oclusão em NSTEMI. }\end{array}$ \\
\hline 21 & WU DF, et al. (2020) & $\begin{array}{l}\text { Relato de caso. Paciente diagnosticado pelo ECG com Síndrome de Wellens. Os resultados sugestivos para } \\
\text { diagnóstico da síndrome foram causas por um fístula da artéria coronária. }\end{array}$ \\
\hline 22 & ZHAN ZQ, et al. (2020) & $\begin{array}{l}\text { Revisão bibliográfica. Compara as diferenças de ECG entre artérias próprias de alterações e várias depressões de } \\
\text { ST (STD). A localização da STD difere entre pacientes com LAD e outras etiologias como padrão de Winter. }\end{array}$ \\
\hline
\end{tabular}

Fonte: Pagoto AB, et al., 2021. 


\section{DISCUSSÃO}

As cardiopatias são um grave problema de saúde mundial, por isso, reconhece-las e trata-las é de fundamental importância. Em condições de emergências, como o Infarto Agudo do Miocárdio (IAM), é crucial ao profissional de saúde saber reconhecer os parâmetros eletrocardiográficos, reconhecendo padrões com elevação ou não do segmento ST. Nessa problemática, sabe-se que muito se fala acerca do IAM com supradesnivelamento do segmento ST, padrão de ECG muito reconhecido e que tem uma terapêutica diferente de outros padrões similares, esses com indicações clínicas parecidas, mas que possuem tratamentos diferentes (PAHLM U, et al., 2014).

Geralmente, o paciente chega ao centro de urgência e emergência com quadro clínico sugestivo de IAM e rapidamente é realizado um ECG, que muitas vezes é avaliado, dentre os diversos padrões que podem ser apresentados. Foram criados sistemas de triagem que têm por função reduzir as demoras no diagnóstico e maior efetividade no tratamento. Infelizmente, tais sistemas ainda estão abaixo do considerado ideal, visto que os profissionais de saúde ainda falham muito no reconhecimento do infarto nos ECGs (AYER A e TERKELSEN CJ, 2014).

Além disso a máxima tempo é miocárdio é uma realidade que precisa ser vivida pelo médico de emergência, uma vez que a demora para manejo do paciente acarreta em incomensuráveis danos na massa muscular cardíaca. Dito isso, evidenciamos em Mclaren JTT, et al. (2020) o tempo médio da chegada do paciente até a realização de ECG como sendo de $9 \mathrm{~min}$ e o tempo médio da interpretação do eletrocardiograma até início da conduta de 16 minutos. Esses dados refletem um tempo hábil na pronta identificação do paciente com dor torácica e solicitação rápida do ECG, mas também coloca a dificuldade do médico em interpretar o exame e iniciar o tratamento de forma acelerada. Isso evidencia falha de cumprimento da máxima, o médico de emergência precisa agir de forma rápida e para isso é importante saber reconhecer o ECG integralmente e com confiança.

Pelas dificuldades anteriores apresentadas e de forma a promover maior acessibilidade ao serviço médico de ECG existem atualmente plataformas para smartphones com disponibilidade de gravação eletrocardiográfica. Esse tipo de tecnologia exclui o limitante da necessidade de um ECG de 12 derivações para fazer diagnóstico de infarto. Segundo o estudo de Muhlestein JB, et al. (2020) para todos os pares de comparação de um ECG usando smartphone e ECG padrão somente 3,9\% foram correlações ruins. Portanto percebe-se que a importância de fazer reconhecimento do ECG vem a se tornar uma necessidade obrigatória do médico a todo médico de emergência.

Sabe-se também que utilizar o eletrocardiograma de 12 derivações é fundamental para o diagnóstico precoce do IAMCSST, visto que suas diferenciações de algumas outras cardiopatias são bem sutis e podem ser ignoradas por médicos inexperientes (HARTMAN SM, et al, 2012). Além disso, é crucial saber que existem diversos padrões de ECG que são caracterizados pela ausência da elevação do segmento ST evidente, eles são chamados de Equivalentes ao IAMCSST, demonstrando sutis mudanças que passam despercebidas por profissionais inexperientes. Isso causa grande prejuízo, acaba levando a falecimentos que poderiam ser evitados, visto que o comprometimento agudo da perfusão coronária requer uma reperfusão imediata que pode ser demorada se dificuldade na percepção do quadro (WINTER RW, et al., 2016).

De acordo com Bosson N, et al. (2017), um dos principais erros de interpretações dos IAM com Supradesnivelamento do segmento ST (IAMCSST) seria artefatos no ECG, que podem levar elevações do segmento ST, mesmo sem representar alguma cardiopatia de fato. Esses artefatos podem estar associados à técnica de realização do exame, com diferenças na disposição dos eletrodos sobre o corpo do paciente, além de fatores intrínsecos ao paciente, como pelos ou músculos.

Sabe-se que não há forma única e inalterável de ensinar aos novos profissionais da saúde a realização e a interpretação dos ECG. No entanto, consideramos que se pode realizar programas de minimização de erros, com análise de diversos exames anualmente. Diversos cursos da área têm duração de poucas semanas e tem um teor prático muito reduzido. De acordo com o American College Of Cardiology e o American Heart Association, o ideal seria a análise de 500 ECGs no período de 12 meses para ser considerado apto a 
diagnosticar, além de 100 ECGs anuais para manter a prática, porém, sabe-se que isso não é realidade em parte dos serviços de saúde, com médicos despreparados para essa habilidade nos serviços de urgência e emergência (BREEN CJ, et al., 2019).

Além disso, existem vários padrões de ECG com alto risco, além do IAMCSST, que podem ser indicativos de oclusão de artéria coronária e o seu reconhecimento é importante, porque impede que hajam atrasos no processo de reperfusão, nesse sentido o reconhecimento desses equivalentes é essencial. Em resumo os padrões equivalentes identificados no ECG são a síndrome de Wellens, sinal de Winter, ondas T hiperagudas, bloqueio de ramo esquerdo e bloqueio de ramo direito (TZIMAS G, et al., 2019).

Entre esses padrões pode-se falar acerca do Padrão de Winter ou Complexo T, que é encontrado em cerca de $2 \%$ dos casos de oclusão aguda da artéria coronária descendente anterior (ADA). A diferenciação seria justamente que o IAMCSST é um fator transitório com ondas T elevadas e simétricas, o que é diferente no Padrão de Winter, que é persistente ao longo do tempo. Tal padrão é importante porque necessita de reperfusão imediata, porém é complicado por sua difícil identificação (CARR M, et al., 2016; YANG W, et al., 2017).

Em Raja JM, et al. (2019) o padrão de Winter mostrou-se raro (próximo de 2\%), mas revelou ser uma patologia grave com alta taxa de mortalidade. É aplicável dissertar também que quase 1 terço dos pacientes que apresentam STEMI tem no mínimo 1 equivalente nas 12 derivações do ECG e o equivalente mais comum é justamente instabilidade em onda T precordial (WISNIEWSKI P, et al., 2019). A artéria descendente anterior costuma ser a artéria culpada e a depressão juncional do segmento ST subindo representa isquemia de maior gravidade do que quando esta não apresenta inclinação (ZHAN ZQ, et al., 2020).

Outra alteração importante a ser pontuada é o Bloqueio de Ramo Esquerdo (BRE) na análise de paciente com possível IAM. Sabe-se que apenas uma minoria dos pacientes com suspeita de síndrome coronariana aguda em conjunto com BRE tem um padrão equivalente IAMCSST. Mas ainda assim as elevações discordantes do segmento ST e ondas T discordantes são fatores preditivos de IAM nesses casos (HANNA EB, et al., 2015). Além disso, sabe-se que os BREs têm maior fator preditor de mortalidade no IAM, ou seja, pacientes que forem diagnosticados precocemente com tal patologia e passarem pela terapêutica adequada rapidamente podem ter um melhor prognóstico.

Em casos em que isso não acontece, a mortalidade se eleva bastante no momento que acontece a obstrução aguda (MACIAS M, et al., 2016). No estudo de Pera VK, et al. (2018) fica claro que paciente com bloqueio de ramo esquerdo é um desafio de investigação, apresentaram bloqueio $3,3 \%$ de um total de pacientes STEMI, além disso esses pacientes representavam uma população de alto risco, ou seja, uma alteração não comum e perigosa.

Para elucidar tal relação do BRE com o IAM, foram criados alguns critérios de seleção quanto à sensibilidade e à especificidade do diagnóstico. O mais famoso seria o Critérios de Sgarbossa (Sgarbossa $\mathrm{EB}$, et al, 1966) que funcionam com a relação do desnivelamento do segmento ST e sua relação com o complexo QRS. Com isso, pacientes que tem pontuação maior ou igual a 3 possuem especificidade de $90 \%$ para a diagnóstico de BRE com IAM sendo fator relevante para a prática médica e na prevenção primária dessas cardiopatias (SEFA N e SAWYER KN, 2016).

Outro padrão de IAM com alta taxa de mortalidade e prognóstico geralmente ruim seria com supradesnivelamento do segmento ST na derivação aVR do ECG. Tal padrão se caracteriza por essa alteração e por infradesnivelamento em quase todas as demais derivações. O fator preocupante no caso é que problemas de IAM nessas regiões são causados por obstrução no tronco coronário, que dá origem às artérias descente anterior e circunflexa, sendo responsável por cerca de $75 \%$ da irrigação do miocárdio no ventrículo esquerdo. Por isso, apesar de se tratar de um quadro raro, tem-se um alto índice de complicações ao paciente e sua má interpretação pelo médico pode levar à morte do doente (KOSUGE M, et al., 2011).

A Síndrome de Wellens é outro padrão eletrocardiográfico relevante. Assim como o Padrão de Winter, possui um carácter duradouro e que, se percebido por um médico experiente, pode evitar um quadro de oclusão aguda posteriormente. A síndrome tem por característica afetar as derivações V2 e V3 (e às vezes 
V4) demostrando carácter bifásico ou invertido Ondas T e afetando a artéria descendente anterior esquerda. Pacientes com essa patologia podem evoluir para um IAM de parede anterior, o que poderia ser prevenido por uma análise de ECG com maior acurácia (LAWNER BJ, et al., 2012). A síndrome é identificada por um padrão específico em que o paciente apresenta ausência de dor torácica com sugestão de oclusão da artéria descendente anterior esquerda ou estenose (WU DF, et al., 2020).

Por fim, podemos inferir que a educação continuada acerca do ECG e dos padrões associados é de extrema importância tanto como fator de prevenção primária de casos quanto de análise para uma terapêutica mais adequada. Sabe-se que médicos e outros profissionais de saúde destreinados podem cometer erros fatais na interpretação do eletrocardiograma, gerando malefícios ao paciente, podendo levar até a morte. Como exemplo, Wei EY, et al. (2013) notaram que muitos critérios diagnósticos de IAMCSST passaram despercebidos nos centros de urgência, seja por desconhecimento dos protocolos acerca do tema ou seja por desatenção ao ECG.

\section{CONSIDERAÇÕES FINAIS}

O IAM com supradesnivelamento do segmento ST é padrão muito reconhecido na interpretação do ECG, no entanto, quanto aos seus equivalentes não podemos afirmar o mesmo. Esses padrões equivalentes são altamente importantes de serem reconhecidos, uma vez que são menos frequentes e apresentam evolução de alto risco para o paciente, além disso a realidade do tempo prolongado de interpretação do ECG pelo médico de emergência é uma evidência da dificuldade atual de reconhecimento desses padrões. Portanto, fica evidente a confirmação da extrema importância do reconhecimento dos equivalentes do supradesnivelamento do segmento ST e a necessidade de que novos estudos sejam elaborados visando um melhor entendimento sobre a temática por parte dos profissionais envolvidos, com base nessa revisão de literatura.

\section{REFERÊNCIAS}

1. AYER A, TERKELSEN CJ. Difficult ECGs in STEMI: Lessons learned from serial sampling of pre-and in-hospital ECGs. Journal of electrocardiology, 2014; 1(1): 448-458.

2. BOND RR, et al. Assessing computerized eye tracking technology for gaining insight into expert interpretation of the 12-lead electrocardiogram: an objective quantitative approach. Journal Of Electrocardiology, 2014; 47(6): 895-906.

3. BOSSON N, et al. Causes of prehospital misinterpretations of ST elevation myocardial infarction. Prehospital Emergency Care, 2017; 21(3): 283-290.

4. BREEN CJ, et al. ECG interpretation skill acquisition: A review of learning, teaching and assessment. Journal of electrocardiology, 2019; 1(1): 1-5

5. CARR MJ, et al. Identification of the STEMI-equivalent de Winter electrocardiogram pattern after ventricular fibrillation cardiac arrest: a case report. The Journal of emergency medicine, 2016; 1(1): 875-880.

6. DE ZWAAN C, et al. Characteristic electrocardiographic pattern indicating a critical stenosis high in left anterior descending coronary artery in patients admitted because of impending myocardial infarction. Am Heart J, 1982; 103(4 Pt 2):730-6.

7. HANNA EB, et al. New or presumably new left bundle branch block in patients with suspected acute coronary syndrome: Clinical, echocardiographic, and electrocardiographic features from a single-center registry. Journal of electrocardiology, 2015; 48(4): 505-511.

8. HARTMAN SM, et al. The use of a 4-step algorithm in the electrocardiographic diagnosis of ST-segment elevation myocardial infarction by novice interpreters. The American journal of emergency medicine, 2012; 30(7): $1282-1295$.

9. KOSUGE M, et al. An Early and Simple Predictor of Severe Left Main and/or Three-Vessel Disease in Patients With Non-ST-Segment Elevation Acute Coronary Syndrome. The American Journal of Cardiology, 2011; $107(4): 495-500$.

10. LAWNER BJ, et al. Novel patterns of ischemia and STEMI equivalents. Cardiology clinics, 2012; 30(4): 591-599.

11. MACIAS M, et al. The electrocardiogram in the ACS patient: high-risk electrocardiographic presentations lacking anatomically oriented ST-segment elevation. The American journal of emergency medicine, 2016; 34(3): 611-617.

12. MCLAREN JTT, et al. Using ECG-To-Activation Time to Assess Emergency Physicians' Diagnostic Time for Acute Coronary Occlusion. The Journal of Emergency Medicine, 2020;1(1): 25-34.

13. MUHLESTEIN JB, et al. Feasibility of combining serial smartphone single-lead electrocardiograms for the diagnosis of ST-elevation myocardial infarction. American heart journal, 2020; 221 (1): 125-135. 
14. PAHLM U, et al. The 24-lead ECG display for enhanced recognition of STEMI-equivalent patterns in the 12-lead ECG. Journal of electrocardiology, 2014; 47(4): 425-429.

15. PERA VK, et al. New or presumed new left bundle branch block in patients with suspected ST-elevation myocardial infarction. European Heart Journal Acute Cardiovascular Care, 2018; 7(3): 208-217.

16. RAJA JM, et al. Is early invasive management as ST elevation myocardial infarction warranted in de Winter's sign-a "peak" into the widow-maker. Annals of translational medicine, 2019; 7(17): 412.

17. SEFA N, SAWYER KN. Smith-modified Sgarbossa criteria and paced rhythms: a case report. The Journal of emergency medicine, 2016; 51(5): 584-588.

18. SGARBOSSA EB, et al. Electrocardiographic diagnosis of evolving acute myocardial infarction in the presence of left bundle-branch block. GUSTO-1 (Global Utilization of Streptokinase and Tissue Plasminogen Activator for Occluded Coronary Arteries) Investigators, N Engl J Med. 1966; 334(7):481-7.

19. SILVA LN, et al. Perfil epidemiológico e clínico de pacientes com síndrome coronariana aguda. Rev enferm UFPE on line, 2018; 12(2): 379-385.

20. SOUZA MT, et al. Revisão integrativa: o que é e como fazer. Einstein (São Paulo). 2010; 8(1): 102-106.

21. TZIMAS G, et al. Atypical electrocardiographic presentations in need of primary percutaneous coronary intervention. The American journal of cardiology, 2019; 124(8): 1305-1314.

22. WALL J, et al. Novel ECG changes in acute coronary syndromes. Would improvement in the recognition of 'STEMIequivalents' affect time until reperfusion? Intern Emerg Med, 2018; 13(2):243-249.

23. WEI EY, et al. Pitfalls in diagnosing ST elevation among patients with acute myocardial infarction. Journal of electrocardiology, 2013; 46(6): 653-659.

24. WINTER RW, et al. Precordial junctional ST-segment depression with tall symmetric T-waves signifying proximal LAD occlusion, case reports of STEMI equivalence. Journal of electrocardiology, 2016; 49(1): 76-80.

25. WINTER RW, et al. Prevalence of junctional ST-depression with tall symmetrical T-waves in a pre-hospital field triage system for STEMI patients. Journal Of Electrocardiology, 2019; 52 (1): 1-5.

26. WIŚNIEWSKI P, et al. Predictive value of electrocardiographic ST-segment elevation myocardial infarction equivalents for detecting acute coronary artery occlusion in patients with non-ST-segment elevation myocardial infarction. Kardiol Pol, 2019; 77(6): 624-631.

27. WISNIEWSKI P, et al. Predictive value of electrocardiographic STEMI-equivalents for detecting acute coronary artery occlusion in patients with non-ST-elevation myocardial infarction. Kardiologia Polska= Polish Heart Journal, 2019; 77(6): 624-631.

28. WU DF, et al. Electrocardiographic findings of Wellens syndrome due to coronary artery-pulmonary artery fistula. Journal of International Medical Research, 2020; 48(5).

29. YANG W, et al. The de Winter electrocardiographic pattern of proximal left anterior descending occlusion. The American journal of emergency medicine, 2017; 35(6): 1095-1096.

30. ZHAN ZQ, et al. The de Winter ECG pattern Distribution and morphology of ST depression. Annals of Noninvasive Electrocardiology, 2020; 25(5): e12783. 